\section{Prácticas espaciales comunes: movilidad de los hogares migrantes pobres en el área central de lquique}

( Pedro Palma Calorio

ONG Observatorio Ciudades Integradas al Territorio CITé, Chile.

\author{
Gricel Labbé Céspedes \\ ONG Observatorio Ciudades Integradas al Territorio CITé, Chile.
}

Recibido: 16 de mayo de 2018. Aceptado: 7 de septiembre de 2018.

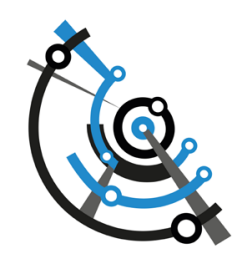

\title{
RESUMEN
}

Los factores decisivos detrás de la elección residencial de un hogar migrante afectan de manera negativa y positiva los diferentes tipos de movilidades que se desenfundan sobre los territorios a través de diversas prácticas espaciales. Cuando estas movilidades presentan dificultades a la hora del desplazamiento, ya sean físicas, sociales, culturales, etc., las prácticas se complejizan. El presente estudio pretende comprender esta realidad desde la elección residencial y la movilidad cotidiana, concentrado el foco de análisis sobre los hogares migrantes latinoamericanos de bajos recursos, y como estos se mueven sobre el espacio, modificando territorios, específicamente en el área central de la ciudad de Iquique. Utilizando notas de campo y entrevistas en profundidad este trabajo analiza el impacto y los costos que tienen las decisiones de localización en la ciudad sobre la experiencia, vida cotidiana de los hogares migrantes pobres.

\section{Common space practices mobility of poor migrant in the central area of lquique}

\footnotetext{
ABSTRACT

The key elements behind the choice of a residential unit by a group or individual, may affect in a positive or negative way the different types of mobility that are carried out over the territories through several spatial practices. When these mobilities present difficulties at the time of moving, either physical (commuting), social, cultural, etc., these practices become complex. The present study hopes to grasp this reality from the choosing of the residendial unit and the quotidian mobility, focusing analysis on low-income Latin American migrant homes, and how they move across the area,
}

Palabras clave

Movilidad cotidiana Prácticas espaciales Migración latinoamericana Área central

Keywords

Quotidian mobility Spatial practices Latin American migration Central area

\section{Palavras-chave}

Mobilidade cotidiana Práticas Espaciais Migração latino-americana Area central 
modifying territories, primarily on the central area of the city of Iquique. Using fieldnotes and in-depth interviews, this work analyses the impact and costs that the choices of location have in the city, regarding the experiences and daily life of the poor migrant homes.

\section{Introducción}

Iquique, ciudad ubicada en el norte de Chile, capital de la región de Tarapacá, es una urbe-puerto y zona franca que cuenta con una población de 191.468 habitantes (Instituto Nacional de Estadísticas INE, 2017). Históricamente ha sido el imán del norte en términos de atractividad para el asentamiento de migrantes. La región de Tarapacá durante los años 1990 y 2004 destacó por su crecimiento debido a las inversiones de la industria del cobre y al dinamismo de la Zona Franca de Iquique (ZOFRI) (Liberona, 2015:22). A raíz de esto, la ciudad se ha ido poblando por diferentes movimientos migratorios, internos como internacionales. ${ }^{1}$ Así lo deja entrever Tapia (2012:183):

La creciente incorporación al mercado mundial a través de la creación de la Zona Franca de lquique, ZOFRI (1975), el fuerte impulso de la actividad minera en la región en las últimas décadas y el impacto de los procesos de integración económica regional, como el MERCOSUR (1991), ha dado lugar a un intenso movimiento de personas y mercancías en el espacio fronterizo del extremo norte. Se trata de un espacio donde existe contacto habitual con las regiones y poblaciones vecinas, lo que se traduce en un intenso trajín fronterizo.

El legado de la migración comienza tempranamente en el siglo XVIII, anterior a la anexión por parte del Estado chileno de la Región, con la llegada de migrantes ingleses, pero también chinos, que dieron a la ciudad la arquitectura y gastronomía que hoy la caracteriza. Posteriormente, durante el siglo XX era común la migración transfronteriza, desde Bolivia, Ecuador y Perú, principalmente ligada a motivos económicos, esto dio origen a antiguos barrios, como el Barrio Esmeralda o San Lorenzo, donde es evidente el sincronismo cultural. Desde la década del 2000 la migración se complejizó en términos de motivaciones, ya no se migraba de forma exclusiva por motivos económicos, sino por refugio, persecución política, huida a la violencia, entre otros, apareciendo nuevos colectivos en la ciudad, sobre todo las vinculadas con la afrodescendencia como dominicanos, colombianos y haitianos.

Hoy, Iquique es una de ciudades que concentra más inmigrantes en Chile en proporción a su población, según las estadísticas del Servicio de Extranjería y Migración (2016), los migrantes provienen en su mayoría de Bolivia (38,8\%), Perú $(31,1 \%)$, y últimamente Colombia (9,2\%) (Instituto Nacional de Estadísticas [INE], 2017) (ver Cuadro 1). Una característica común de estos grupos es que son hogares de bajos ingresos que tienden a ubicarse preferentemente en el centro y pericentro de la ciudad, esto último por las posibilidades de acceso a una vivienda barata, que ha ido consolidando diversos enclaves étnicos en centro de la ciudad.

Cuadro 1. Porcentaje de colectivos respecto al total de inmigrantes en Iquique. Fuente: Elaboración propia a partir Censos de Vivienda y Población.

\begin{tabular}{|l|c|c|c|c|c|c|c|c|}
\hline Inmigrantes & Argentina & Perú & Bolivia & Colombia & Ecuador & Venezuela & Haití & Otros \\
\hline 1992 & 14,2 & 19,3 & 22,7 & 0,8 & 2,7 & 0,6 & 0,2 & 39,1 \\
\hline 2002 & 6,1 & 40,2 & 21,6 & 0,6 & 5,1 & 0,7 & 0,01 & 25,3 \\
\hline 2017 & 2,3 & 31,3 & 38,8 & 9,2 & 3,3 & 1,8 & 0,5 & 12,6 \\
\hline
\end{tabular}

1. Cabe destacar que Iquique siempre ha estado sometida a migración transfronteriza, es por esto, que no es extraño observar en la época salitrera migrantes peruanos, bolivianos, e inclusive europeos trabajando en la industria. 
Liberona (2015:21) plantea que el fenómeno de la migración se ha masificado desde la década del 1990 y ha alcanzado particularidades en la Región, marcado fuertemente por una impronta de migración andina:

Ya en el censo de 2002, la Región de Tarapacá se presentaba como la primera con mayor inmigración relativa, con 6,66\% de población inmigrante en relación al total de su población. Los datos del censo de 2012 indican que este porcentaje ha aumentado a 7,4\%. La población boliviana representa 32,2\% y es la segunda con mayor representación en la región.

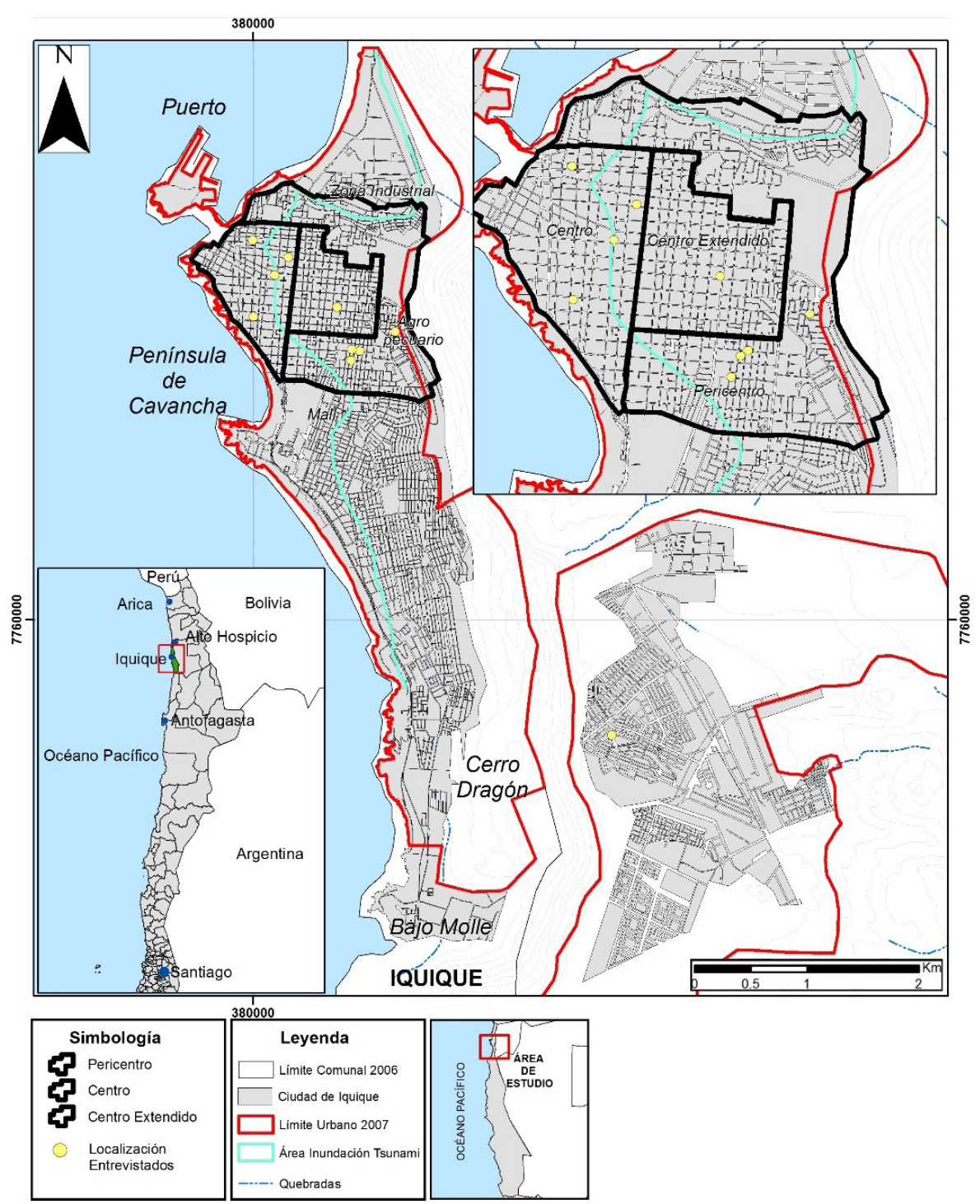

Figura 1. Área de estudio: El área central de Iquique. Fuente: Elaboración cartográfica a partir de datos del Fondecyt $N^{\circ} 11121241$.

El motivo detrás de la localización de los migrantes en el área central de Iquique es la búsqueda de proximidad a sus redes sociales y familiares, por sobre la cercanía al trabajo (Palma, 2014). Al respecto, Tapia (2012) asegura que los migrantes vienen a ocupar nichos laborales que hoy la población nacional considera riesgosos y de baja cualificación. Estás fuentes de trabajos se localizan, por un lado, al sur de la ciudad (Figura 1) correspondiente al sector de Bajo Molle, al norte próximos a la Zona Franca de Iquique (ZOFRI) y, por último, hacia comunas aledañas por la extracción de minerales. 
Los migrantes se ven obligados a viajar diariamente hacia áreas alejadas de la residencia, lo que provoca un aumento en la distancia y tiempo de los commuting (desplazamientos) complejizando el proyecto migratorio como también las prácticas espaciales. Del planteamiento anterior cabe preguntarse entonces ¿Cuáles son las prácticas espaciales de los nuevos migrantes, y qué relación existe entre la movilidad cotidiana laboral de los hogares migrantes y su elección residencial? Para ello se examinarán las prácticas espaciales y el vínculo con la elección residencial central.

Metodológicamente, para el análisis se utilizaron técnicas cualitativas, las cuales hacen referencia a la aplicación de entrevistas en profundidad a 10 hogares migrantes latinoamericanos ${ }^{2}$ extraídos de una encuesta realizada por el Fondecyt $\mathrm{N}^{\circ} 11121241$, empleada a 136 hogares de bajos ingresos situados en el centro y pericentro de la ciudad de Iquique ${ }^{3}$ (Figura 1).

\section{Movilidad, migración y ciclo vida}

El estudio de la migración remite al estudio de la movilidad en función del ciclo de vida de familias migrantes, como marco interpretativo de las decisiones y los factores que intervienen a la hora de acceder a una ubicación dentro del conglomerado urbano. La movilidad sería así un factor de explicación de los procesos urbanos y de los comportamientos sociales dentro de la ciudad (Dureau, 2007) y que corresponde al proceso sucesivo o reiterado, que busca optimizar la combinación de los parámetros que determinan la localización residencial. Dentro de estos parámetros, se destaca el "ciclo de vida" en el cual se ve representado en la conformación familiar, y es uno de los principales elementos de análisis, ya que cada etapa está asociada a diferentes eventos que modifican las necesidades y preferencias de vivienda, y que pueden verse afectado por las tendencias de cambios demográficos en la población (Garzón, 2010:13).

Entonces, la elección residencial se centra en los elementos que se relacionan con estos parámetros, los cuales están dados por las características de la familia, del inmueble deseado y su entorno, además influye en los procesos de urbanización de las diferentes ciudades o zonas metropolitanas siendo fuerte factor de conformación de la ciudad como lo dejan entrever Ortiz y Schiappacasse (1997).

\section{Espacios de vida y prácticas espaciales}

La movilidad internacional implica cambios en el espacio de vida que se observan en las transformaciones de las prácticas y representaciones urbanas. "Una forma como estas dimensiones se funden con la movilidad y fundamentalmente con las dinámicas residenciales son los conceptos de espace de vie (espacio de vida) por un lado, y el modo de vida, por el otro" (Córdoba, 2014:76). El primero según Fremont (en Córdoba, 2014) se refiere al conjunto de lugares frecuentados. Este término va de la mano con el de espace vécu (espacio vivido) propuesto por el mismo autor, el cual no se limita a reconocer lugares frecuentados, definir itinerarios, situar al hombre-habitante en su cuadro familiar de existencia, sino focalizar la mirada en la relación con las representaciones. "El espacio vivido es reivindicado como un espacio cargado de valores" (Hiernaux y Lindón, 2006: 382).
2. Las nacionalidades entrevistadas representan el mayor número de migrantes en la ciudad, estos son Perú, Bolivia, Colombia y Ecuador respectivamente. La identidad de cada entrevistado se encuentra protegida, asignando el apelativo de 'caso' para cada descripción.

3. Se realizaron 307 encuestas en el Proyecto Fondecyt $\mathrm{N}^{\circ}$ 11121241. Para adquirir mayor detalle sobre la metodología aplicada y resultados obtenidos visitar el link http://repositorio. uchile.cl/handle/2250/129886. 
Para Córdoba (2014), en el desplazamiento de un país a otro, los migrantes internacionales pueden desarrollar estrategias residenciales que implican o no cambios en la movilidad residencial. Este cambio caracterizado por la toma de la distancia espacial puede llevar a la reconfiguración del espacio de vida en los dos extremos espaciales. "En otras palabras las formas de habitar el lugar residencial actual serían influenciados por las diferencias entre los dos espacios de vida y por la distancia que los separa" (Dubucs, 2009:56). Entonces, según (Dupont \& Dureau, 1994) el espacio de vida corresponde al espacio de las prácticas sociales de un individuo. Esta noción permite tomar en cuenta el carácter múltiple de la localización del individuo en el espacio.

En el caso de la movilidad internacional, el cambio que se genera en los espacios de vida y en las representaciones, se consideran en la medida que transforman al sujeto migrante con las distintas experiencias migratorias. Según Córdoba (2014:77);

Estas mismas representaciones sobre el espacio y los lugares movilizan determinadas prácticas urbanas y la escogencia residencial, cuya atención se ha prestado en el caso de los migrantes internacionales en el momento en que se enfrentan con lugares inéditos al momento de la llegada.

En muchos casos, los migrantes a su llegada habitan en condición de hacinamiento o en viviendas deterioradas, debido a que buscan satisfacer las necesidades básicas (techo, comida, trabajo, educación) en la primera etapa del proyecto migratorio, en la cual según Llano (2005) se vincula la residencia con el lugar de trabajo. La noción toma en cuenta las características del desplazamiento hacia el lugar de trabajo, la especificidad del sitio frecuentado (estatus público o privado, localización, función principal) y la actividad realizada en él (Marcadet, 2007).

\section{Los casos de estudio}

De las entrevistas realizadas se desprende que existe una relación directa entre la etapa que está cursando el inmigrante y la estabilidad del proyecto migratorio, ya sea en términos, laborales, residenciales u otros. Otro punto a destacar es que a pesar de la etapa del ciclo, ya sea de estabilidad (la cual se caracteriza inicialmente por la capacidad desarrollada por el inmigrante para participar en el nuevo entorno, tratando de introducirse a las nuevas formas sociales a través de la localización y uso de nuevos espacios de encuentro con compatriotas y locales) o de transformación (que se caracteriza por la decisión de retornar en un corto plazo al país de origen o por otro lado radicarse definitivamente en el país receptor,) según Granados (2011), las prácticas espaciales de estos hogares migrantes tienden a ser las mismas, independiente de la condición histórica (migración de los años 90, peruana, boliviana, ecuatoriana) o nueva (colombiana, venezolana, haitiana).

Según lo anterior, se procedió a clasificar a los migrantes de acuerdo a cuatro variables; 1) trayectoria residencial y espacios habitados, 2) Etapa en la línea de vida (Granados, 2011), 3) Criterio de elección residencial y 4) Retorno. En base a esto las tres categorías resultantes son; Inmigrantes transitorios, permanentes e inciertos. Si bien el análisis va en línea con la respuesta de los entrevistados, es posible que las categorías puedan representar las dinámicas de una nacionalidad, como también es de esperarse que algunas categorías concentren más de una nacionalidad (Cuadro 2). 
Tabla 2. Categorías entrevistados. Fuente: Elaboración propia de entrevistas en profundidad. Palma (2013).

\begin{tabular}{|l|c|c|c|}
\hline Rasgo diferente & Migrantes transitorios & $\begin{array}{c}\text { Migrantes } \\
\text { permanentes }\end{array}$ & Migrantes inciertos \\
\hline $\begin{array}{l}\text { Trayectoria residencial } \\
\text { y espacios habitados }\end{array}$ & $\begin{array}{c}\text { Son multimóviles en } \\
\text { el país de origen y lo } \\
\text { replican en el país de } \\
\text { destino. Los cambios } \\
\text { residenciales están } \\
\text { asociados a conflictos } \\
\text { matrimoniales y } \\
\text { búsqueda de trabajo. } \\
\text { Hogares precarios. }\end{array}$ & $\begin{array}{c}\text { Movilidad se } \\
\text { incrementa en el país } \\
\text { de destino a nivel } \\
\text { intraurbano. Cambios } \\
\text { residenciales están } \\
\text { asociados a crisis } \\
\text { económicas. }\end{array}$ & $\begin{array}{c}\text { Son multimóviles en } \\
\text { el país de origen como } \\
\text { de destino. Hogares } \\
\text { arrendatarios, tiempo } \\
\text { de estancia es incierto. } \\
\text { Es el grupo más } \\
\text { vulnerable. }\end{array}$ \\
\hline $\begin{array}{l}\text { Tiempo y espacio en } \\
\text { la línea de vida }\end{array}$ & $\begin{array}{c}\text { Etapa de adaptación } \\
\text { Retarno }\end{array}$ & $\begin{array}{c}\text { Estabilidad y } \\
\text { transformación }\end{array}$ & $\begin{array}{c}\text { Transitan de la } \\
\text { adaptación a la } \\
\text { estabilidad }\end{array}$ \\
\hline $\begin{array}{l}\text { Criterio de elección } \\
\text { residencial }\end{array}$ & $\begin{array}{c}\text { El acceso y cercanía } \\
\text { a sus redes sociales y } \\
\text { familiares }\end{array}$ & $\begin{array}{c}\text { Cercanía a espacios de } \\
\text { servicios }\end{array}$ & Acceso arriendo \\
\hline Retorno & $\begin{array}{c}\text { Retornan esporádica y } \\
\text { continuamente al país } \\
\text { de origen }\end{array}$ & $\begin{array}{c}\text { No retornan más que } \\
\text { para visitar familiares } \\
\text { y/o parientes }\end{array}$ & $\begin{array}{c}\text { Esperan retornar a un } \\
\text { largo plazo }\end{array}$ \\
\hline
\end{tabular}

Las categorías de migrantes aquí presentadas son dependientes a las estructuras sociales, políticas y económicas en las que están inmersos, indistintamente de su condición de migrante. Estas clases a su vez, son dependientes del espacio y tiempo en los que se inscribe el proyecto migratorio. En un sentido estricto, el espacio predetermina los sitios de acceso del migrante, en tanto el tiempo, está vinculado al proyecto migratorio del sujeto. De ahí que se apela a la circularidad en las categorías de los migrantes: un migrante permanente puede pasar a una condición de transitoriedad e incertidumbre o viceversa en la medida en que existan cambios en las estructuras sociales, políticas y económicas que redefinen esa condición.

De acuerdo a las 10 entrevistas realizadas, 3 corresponden a migrantes transitorios, 4 a migrantes inciertos, y 3 a migrantes permanentes (Cuadro 3 ).

Cuadro 3. Inmigrantes Entrevistados. Fuente: Elaboración propia de entrevistas en profundidad. Palma (2013).

\begin{tabular}{|l|c|c|c|c|}
\hline Colectivo & $\begin{array}{c}\text { Llegada a } \\
\text { lquique }\end{array}$ & Trayectoria residencial y espacio habitado & Motivos Migración & Categoría \\
\hline $\begin{array}{l}\text { Hogar migrante } \\
\text { boliviano 1.1 }\end{array}$ & 2011 & $\begin{array}{c}\text { Múltimóvil en el país de origen y des tino. Menor } \\
\text { tiempo de estancia en las residencias. }\end{array}$ & $\begin{array}{c}\text { Quiebre matrimonial } \\
\text { (engaño del } \\
\text { esposo). }\end{array}$ & TRANSITORIO \\
\hline $\begin{array}{l}\text { Hogar migrante } \\
\text { boliviano 1.2 }\end{array}$ & 2010 & $\begin{array}{c}\text { Múltimóvil en el país de origen y destino. El } \\
\text { tiempo de estancia varía dependiendo del criterio } \\
\text { de arriendo que considere. }\end{array}$ & $\begin{array}{c}\text { Quiebre matrimonial } \\
\text { (agresión física y } \\
\text { psicológica del } \\
\text { esposo). }\end{array}$ & INCIERTO \\
\hline $\begin{array}{l}\text { Hogar migrante } \\
\text { boliviano 1.3 }\end{array}$ & 2013 & $\begin{array}{c}\text { Múltimóvil en el país de origen y des tino. Menor } \\
\text { tiempo de estancia en las residencias. }\end{array}$ & Laboral. & TRANSITORIO \\
\hline $\begin{array}{l}\text { Hogar migrante } \\
\text { colombiano 1.4 }\end{array}$ & 2007 & $\begin{array}{c}\text { Múltimóvil en el país de origen y des tino. El } \\
\text { tiempo de estancia varía dependiendo del criterio } \\
\text { de arriendo que considere }\end{array}$ & $\begin{array}{c}\text { Laboral y crisis } \\
\text { económica. }\end{array}$ & INCIERTO \\
\hline $\begin{array}{l}\text { Hogar migrante } \\
\text { colombiano 1.5 }\end{array}$ & 2007 & $\begin{array}{c}\text { Múltimóvil en el país de origen y des tino. El } \\
\text { tiempo de estancia varía dependiendo del criterio } \\
\text { de arriendo que considere }\end{array}$ & Laboral y crisis \\
económica. & INCIERTO \\
\hline $\begin{array}{l}\text { Hogar migrante } \\
\text { colombiano 1.6 }\end{array}$ & 2012 & $\begin{array}{c}\text { Múltimóvil en el país de origen y des tino. El } \\
\text { tiempo de estancia varía dependiendo del criterio } \\
\text { de arriendo que considere }\end{array}$ & Laboral y crisis \\
económica. & INCIERTO \\
\hline
\end{tabular}




\begin{tabular}{|l|c|c|c|c|}
\hline $\begin{array}{l}\text { Hogar migrante } \\
\text { ecuatoriano 1.7 }\end{array}$ & 2011 & $\begin{array}{c}\text { Múltimóvil en el país de origen y des tino. Menor } \\
\text { tiempo de estancia en las residencias }\end{array}$ & $\begin{array}{c}\text { Problemas económicos } \\
\text { en Ecuador. Pagar una } \\
\text { deuda. }\end{array}$ & TRANSITORIO \\
\hline $\begin{array}{l}\text { Hogar migrante } \\
\text { ecuatoriano 1.8 }\end{array}$ & 2008 & $\begin{array}{c}\text { Presenta pocos cambios en el país de origen, pero } \\
\text { es tos aumentan levemente en el país de des tino. } \\
\text { El tiempo de estancia es más prolongado. }\end{array}$ & $\begin{array}{c}\text { Para no perder el } \\
\text { pasaje que utilizaría } \\
\text { para viajar a Italia. }\end{array}$ & PERMANENTE \\
\hline $\begin{array}{l}\text { Hogar migrante } \\
\text { peruano 1.9 }\end{array}$ & 1997 & $\begin{array}{c}\text { Presenta pocos cambios en el país de origen, pero } \\
\text { es tos aumentan levemente en el país de des tino. } \\
\text { El tiempo de estancia es más prolongado. }\end{array}$ & Laboral. & PERMANENTE \\
\hline $\begin{array}{l}\text { Hogar migrante } \\
\text { peruano 2.0 }\end{array}$ & 1989 & $\begin{array}{c}\text { Presenta pocos cambios en el país de origen, pero } \\
\text { es tos aumentan levemente en el país de destino. } \\
\text { El tiempo de estancia es más prolongado }\end{array}$ & $\begin{array}{c}\text { Llega por un evento } \\
\text { a la Zofri, crisis } \\
\text { económica impulso } \\
\text { quedarse. }\end{array}$ & PERMANENTE \\
\hline
\end{tabular}

\section{Prácticas espaciales comunes en lquique}

De las entrevistas en profundidad se concluye que la elección por un determinado sitio dentro de la ciudad está medida por un trade off entre diferentes "aspectos materiales y objetivos a los que se suman las características individuales y familiares, las trayectorias biográficas, entre otros" (Contreras, 2012:223). Los factores en la elección residencial vienen a establecer las características y la intensificación de la creciente movilidad cotidiana, o como plantea Rodríguez (2008) la creciente conmutación, que involucra un mayor número y una mayor distancia de desplazamientos, por motivos de trabajo, estudio y compras, entre otras actividades.

Es así como la exploración de los datos recopilados proporcionó información relevante sobre la movilidad cotidiana fundada en las prácticas espaciales, lo que permitió vincular el motivo de elección residencial con los diferentes tipos de desplazamientos en la ciudad y como estos despliegan sus prácticas espaciales a partir de sus preferencias y además de acuerdo a la etapa del ciclo migratorio en el que se encuentren. En la descripción a continuación se presentan cinco casos.

\section{¿Cercanía o lejanía al trabajo? Una decisión que afecta el commuting de los hogares migrantes pobres}

La localización residencial próxima al área de servicios y trabajo permite que el capital de movilidad de los hogares migrantes pobres se vea favorecido, como así también la proximidad a las redes sociales, ya que tienden a coincidir espacialmente estos tres criterios (fuente laboral, comercio, familia y amistades), a pesar de que para algunos hogares migrantes la cercanía a las redes no es una prioridad a la hora de la localización.

Un ejemplo de lo anterior son los entrevistados 1.8, 1.9 y 2.0, estos hogares presentan un patrón en común que dista del uso del área central (centro, centro extendido y pericentro) en su totalidad. A pesar de esto, se observa que los migrantes no se movilizan por la extensión total de la urbe, es decir, existe una movilidad acotada al centro de la ciudad, que coincide con barrios de migración histórica.

Un caso representativo es el 2.0 (Figura 2), el cual escoge vivir cerca del centro por encontrarse próximo a los servicios que el centro confiere. Los desplazamientos semanales que corresponden al trabajo de él y su esposa, los realizan en taxi en un promedio entre 5 a 10 minutos, en el caso de él a la ZOFRI (soldador) y de ella al Retail (secretaria). Los desplazamientos del fin de semana son realizados principalmente a pie, independiente del motivo por compras u ocio. 


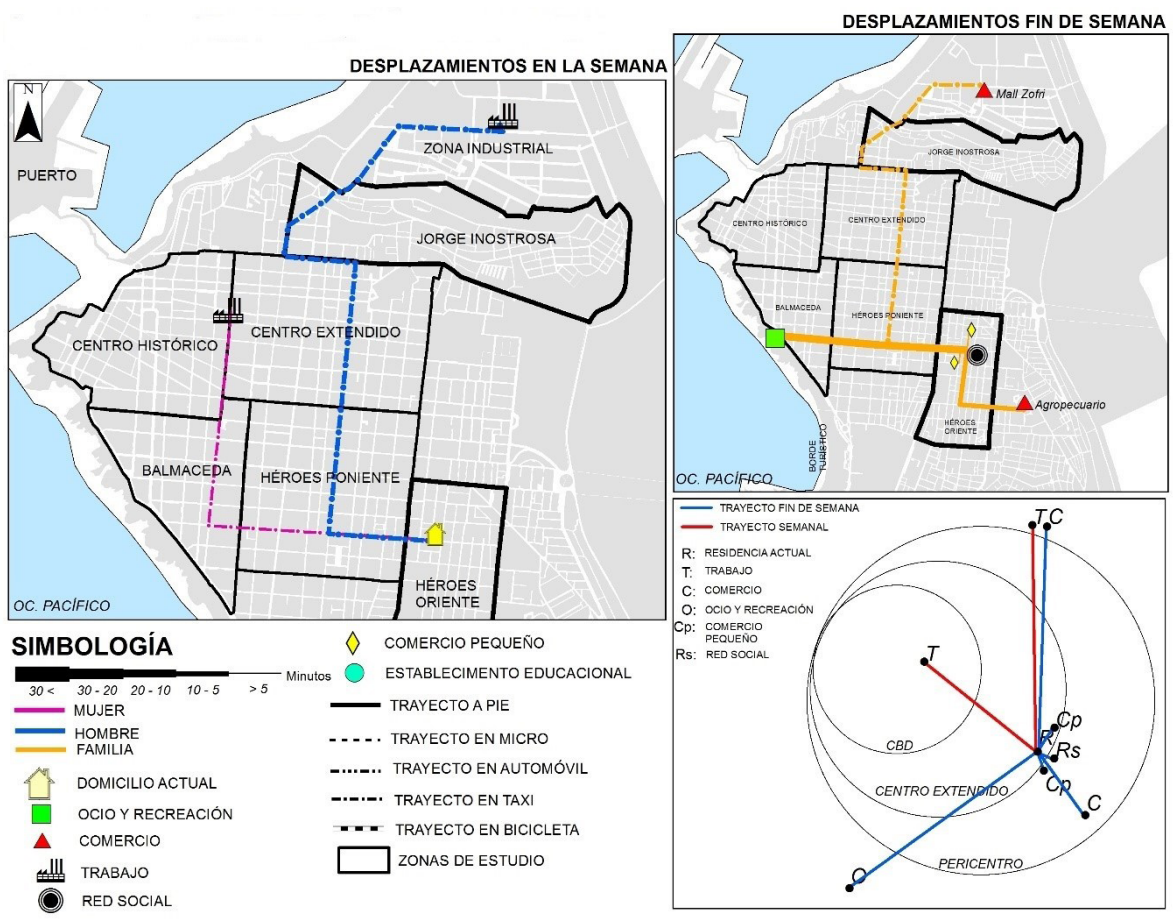

Figura 2. Hogar migrante peruano 2.0, permanente. Fuente: Elaboración propia a partir de las entrevistas en profundidad Fondecyt $N^{\circ} 11121241$.

La Figura 2 corrobora que existe un predominio de movimientos próximos a la residencia actual del hogar. Se observa además que, a pesar de la estabilidad del proyecto migratorio, las prácticas espaciales son similares como se mostrará a continuación con otros coterráneos, estando limitadas al área central. Lo interesante a rescatar es que existe un ahorro sustantivo en todos los desplazamientos generados que se verían plasmados en la economía del hogar, así lo deja ver la entrevista;

Aquí me queda todo cerca, los sábados en la mañana voy al agropecuario, luego llevo a mi hijo a la playa a pescar y todo lo hago caminando, así me ahorro pesitos para la semana si necesito movilizarme más de la cuenta por el trabajo (hogar migrante; jefe de hogar, 64 años)

Esta condición da un vuelco distinto a la hora de enfrentar la lejanía al trabajo. Existe un aumento del tiempo del desplazamiento laboral cuando la fuente de trabajo se encuentra fuera del área central, en las secciones periféricas u otras comunas. Independiente del modo usado para el desplazamiento, el promedio de tiempo de traslado es de 30 minutos, así se observa en el caso 1.5 y 1.7, este último caso (Figura 3 ) es el más significativo ya que presenta la disyuntiva de haber escogido la residencia por encontrarse el trabajo próximo, pero la volatilidad laboral generó que su trabajo hoy se encuentre fuera del radio que ella considera cercano, así lo describe en la entrevista:

Ahora estoy trabajando en la zona sur hacia Bajo Molle, me ha sido muy pesado el cambio de trabajo hacia allá, porque antes hacía aseo en una casa aquí cerca por eso también arrendé en este sector. Ahora me demoro 30 minutos en bus y en taxi un poco menos, pero antes me demoraba menos de 10 caminando (hogar migrante; jefa del hogar, 28 años). 


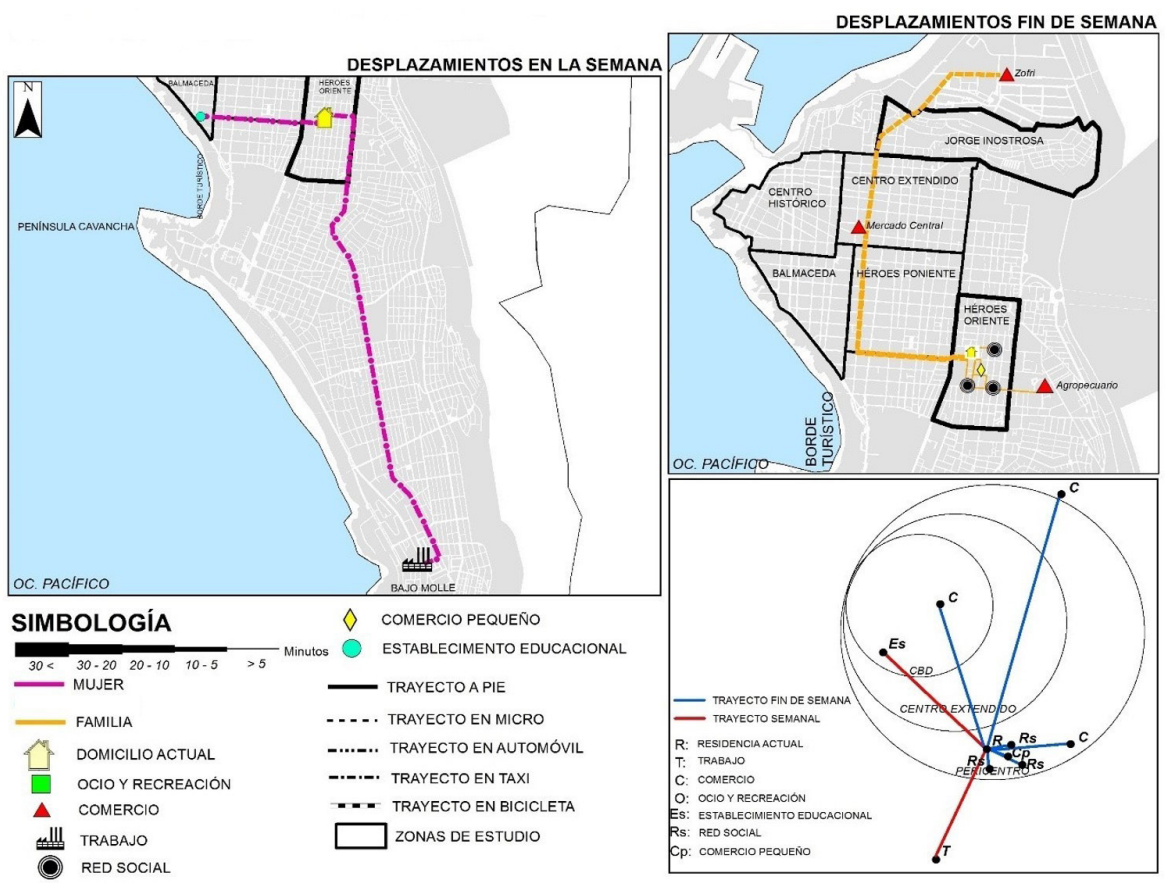

Figura 3. Hogar migrante ecuatoriano 1.7, transitorio. Fuente: Elaboración propia a partir de las entrevistas en profundidad Fondecyt $N^{\circ} 11121241$.

En este caso se presenta la dualidad de buscar la residencia próxima a la fuente laboral versus el cambio de trabajo hacía el exterior del espacio transitado. El caso 1.5, donde la jefa era asesora del hogar y trabajaba en el sector Héroes poniente en una vivienda, como asesora "puertas afuera", el desplazamiento lo realizaba a pie en un promedio de 5 minutos. Con el cambio de trabajo el tiempo y la distancia de este desplazamiento se vieron alterados ya que su fuente laboral se encuentra en Bajo Molle, al cual llega en taxi y se demora en promedio 10 a 20 minutos en llegar. Con ello se intensificó el uso de la proximidad como capital de movilidad para disminuir los costos asociados al aumentar el tiempo y distancia de los commuting. Por ende, las compras que realizaba semanalmente en la ZOFRI, fueron reemplazadas por desplazamientos hacia el Mercado del Agro y hacia negocios del barrio.

\section{Cercanía a redes sociales y familiares. Proximidad en la movilidad cotidiana.}

Del universo de entrevistados (10), 8 habitan en viviendas sub-arrendadas (familiar o de amigos) en zonas en transformación y deterioro. Generalmente comparten los servicios básicos como el baño y la cocina. Estos migrantes son aquellos que aún no se encuentran en un período de estabilidad, por ende, el costo en términos de desplazamientos laborales que asumen por encontrar su vivienda en el centro está asociado a la proximidad de sus redes.

El valor por habitar cerca de sus redes sociales, especialmente para los migrantes latinoamericanos es significativo, ya que disminuyen una serie de costos asociados al proyecto migratorio. Las redes proporcionan o ayudan en la búsqueda de alojamientos y trabajos, a veces como es el caso 1.1 y 1.6 sus trabajos coinciden con la cercanía a las redes. Según Stefoni (2002:34) la red es un "conjunto de vínculos interpersonales que conectan a migrantes, antiguos migrantes y no migrantes en su área de origen y de destino a través de los lazos de parentesco, amistad y comunidad de origen compartida". 
En relación con los beneficios que otorgan las redes a la hora de disminuir los costos de desplazamientos, estos van más bien asociados al tránsito hacia el sector de compras, educacional, etc. Para las compras diarias o semanales sobre todo de alimentos, los entrevistados recurren a supermercados ubicados dentro del radio en el cual habitan, disminuyendo así en costo y tiempos de traslado, ya que pueden llegar hasta estos lugares caminando. Si bien hacen un uso de proximidad con su barrio concurren al capital comercial moderno que se instala dentro o próximo a su lugar de residencia. Ejemplo de esto es el estudio de caso 1.6 (Figura 4).

Estudios empíricos como los realizados por Ávila (2015:163) en Barcelona, a través de la utilización de encuestas que cuantificaban las relaciones y redes sociales entre migrantes, evidenció que el 91\% de los migrantes (266) tenían redes de reciprocidad transnacional con sus países de origen. En su mayoría los migrantes se relacionan con gente de su misma nacionalidad.

En términos generales los datos muestran que los migrantes tienen redes de reciprocidad en su mayoría con personas de su misma nacionalidad de origen, en un rango que varía entre el $53,54 \%$ (senegaleses) y el $81,3 \%$ (dominicanos). Las redes de reciprocidad con españoles son menores, en un rango entre el $15,46 \%$ (dominicanos) y el 29,03\% (argentinos). Finalmente, las redes de reciprocidad con personas de otras nacionalidades tienen un rango entre el 2,24\% (marroquíes) y el $30,12 \%$ (senegaleses).

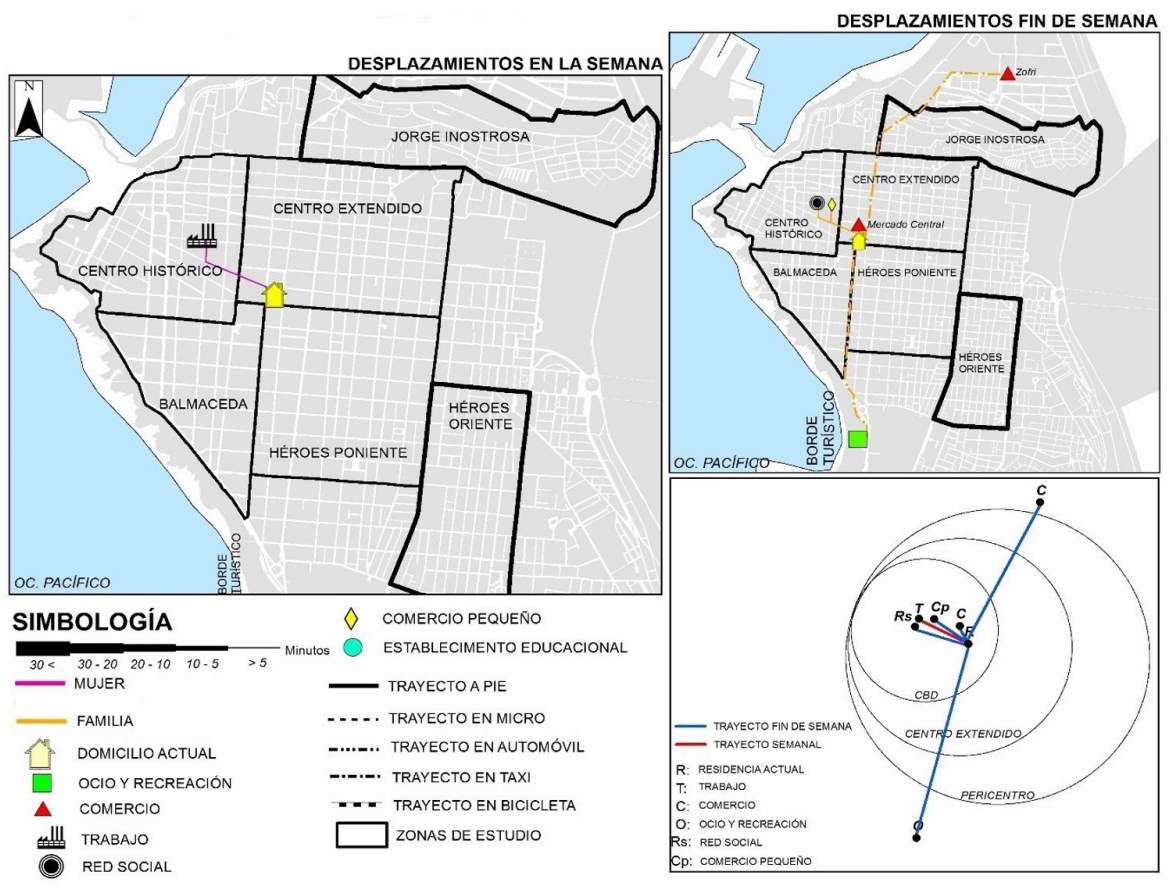

Figura 4. Hogar migrante colombiano 1.6, incierto. Fuente: Elaboración propia a partir de las entrevistas en profundidad Fondecyt $N^{\circ} 11121241$.

En el caso 1.6, la jefa del hogar es peluquera y cada fin de semana presenta una movilidad cotidiana de proximidad, ya que las compras domésticas las realiza en pequeños locales en el mismo barrio. Solo sale del área central hacia la ZOFRI, pero esto ocurre esporádicamente, una vez al mes.

Otro es el caso 1.5 (Figura 5), quien trabaja en la misma vivienda con un emprendimiento en una empresa de exportación de ropa de cama, junto a su marido se dirigen a 
la ZOFRI una vez por semana en búsqueda de materia prima con la que confeccionan la ropa de cama que exportan. Como familia se desplazan para sus compras en el mismo barrio y solo salen de este para asistir a la ZOFRI o el Mall las Américas, en el sector sur de la ciudad, a 15 minutos en vehículo del centro. De la entrevista se rescata lo siguiente:

Hago mis compras por acá por el sector, visito a mis amigas, voy al mall, casi siempre caminando solo a veces tomo un taxi de vuelta o viajo en mi moto (hogar migrante; jefa del hogar, 49 años).

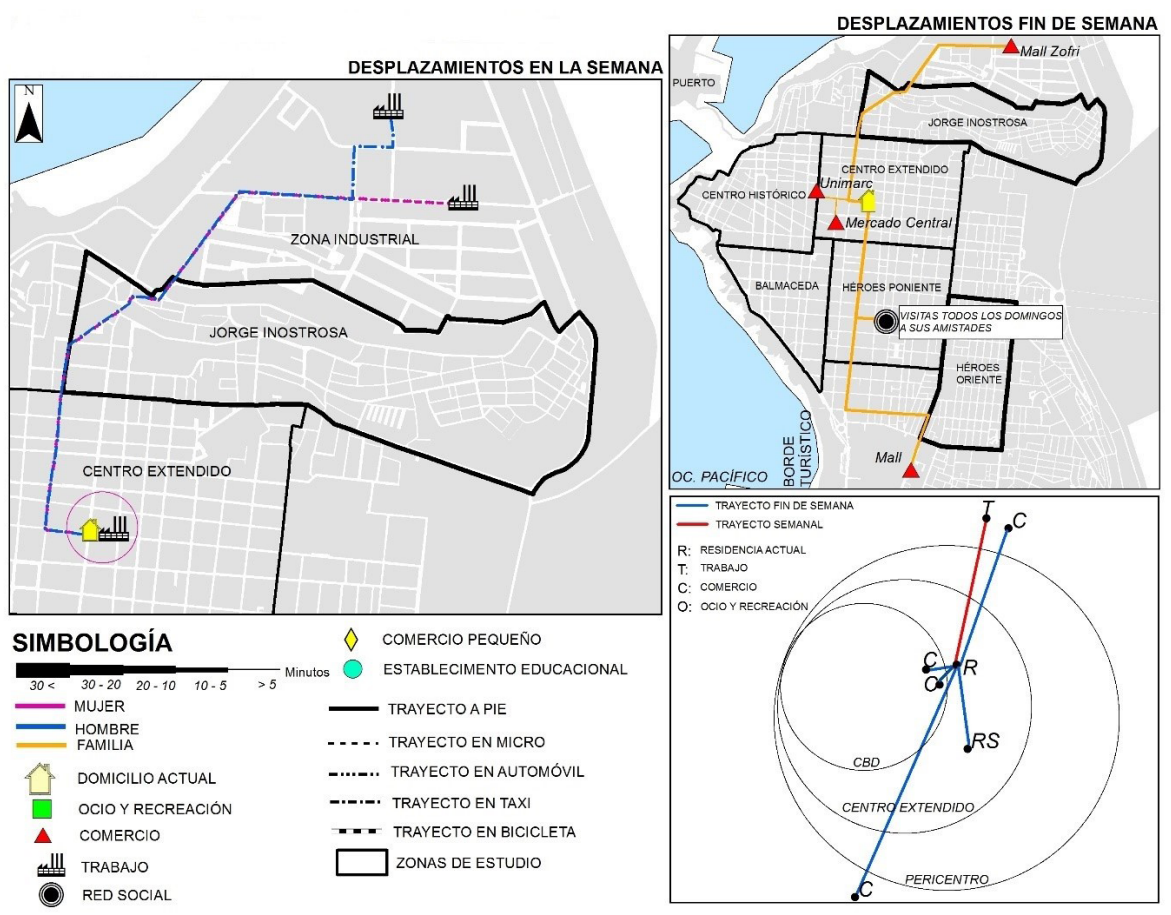

Figura 5. Hogar migrante colombiano 1.5, incierto. Fuente: Elaboración propia a partir de las entrevistas en profundidad Fondecyt $N^{\circ} 11121241$.

Se observa que las prácticas de proximidad surgen como un recurso que diversifica la posibilidad de movilidad. Estas prácticas tanto semanales como de fin de semana hablan de cambios en la relación espacio-tiempo, expresada en una movilidad de proximidad durante el fin de semana y una apertura al exterior asociada al ocio y compras mensuales en mercados y mall, debido que, en la periferia el espacio urbano ha sido moldeado en base a equipamientos urbanos anclas, como centros comerciales, strip center y paseos peatonales.

Esto último, según Orfeuil (2004) en Contreras (2012) asemeja una aptitud a la movilidad, es decir, pese a la condición de vulnerabilidad económica, social o familiar, temporal o permanente, ellos tienen una aptitud, habilidad o tradición de ser móviles en aquellos sitios que definieron sus trayectorias y recorridos residenciales, sociales y familiares.

Estas prácticas espaciales se encuentran concentradas en aquellos sitios donde habitan sus redes sociales y familiares, lo cual convierte al barrio o población en anclas y nodos a partir de los cuales articulan sus movilidades, siendo la vivienda el pivote principal que asegura las prácticas.

Un caso especial es el 8 (hogar migrante; jefa del hogar, 48 años) (Figura 6), hogar boliviano que logró acceder al arriendo de una vivienda en la comuna de Alto Hospicio y aun así el vínculo laboral y educacional con Iquique no se pierde. 
La elección de ubicarse cercana a sus redes y la opción de un arriendo más barato generó que el tiempo de traslado por motivos laborales haya aumentado considerablemente de cuando vivía en el pericentro de Iquique. Esto se vería intensificado post terremoto 2014, el cual provocó problemas en la vía que conecta dicha comuna con Alto Hospicio, hoy en día el tiempo de traslado es al menos de 2 horas:

Después de trabajar en el club Cavancha, logré encontrar trabajo en el aeropuerto, el cual coincidió con él cambio de casa acá a Alto Hospicio donde le arriendo a mi amiga. El único problema de este cambio es que llegó muy tarde a mi casa y me debo levantar muy temprano para el trabajo porque me demoró mucho en llegar y entro a las 8, imagínese a las 6 debo estar saliendo a tomar la micro y si no pasa debo pagar un colectivo hasta Iquique (hogar migrante; jefa del hogar, 48 años).

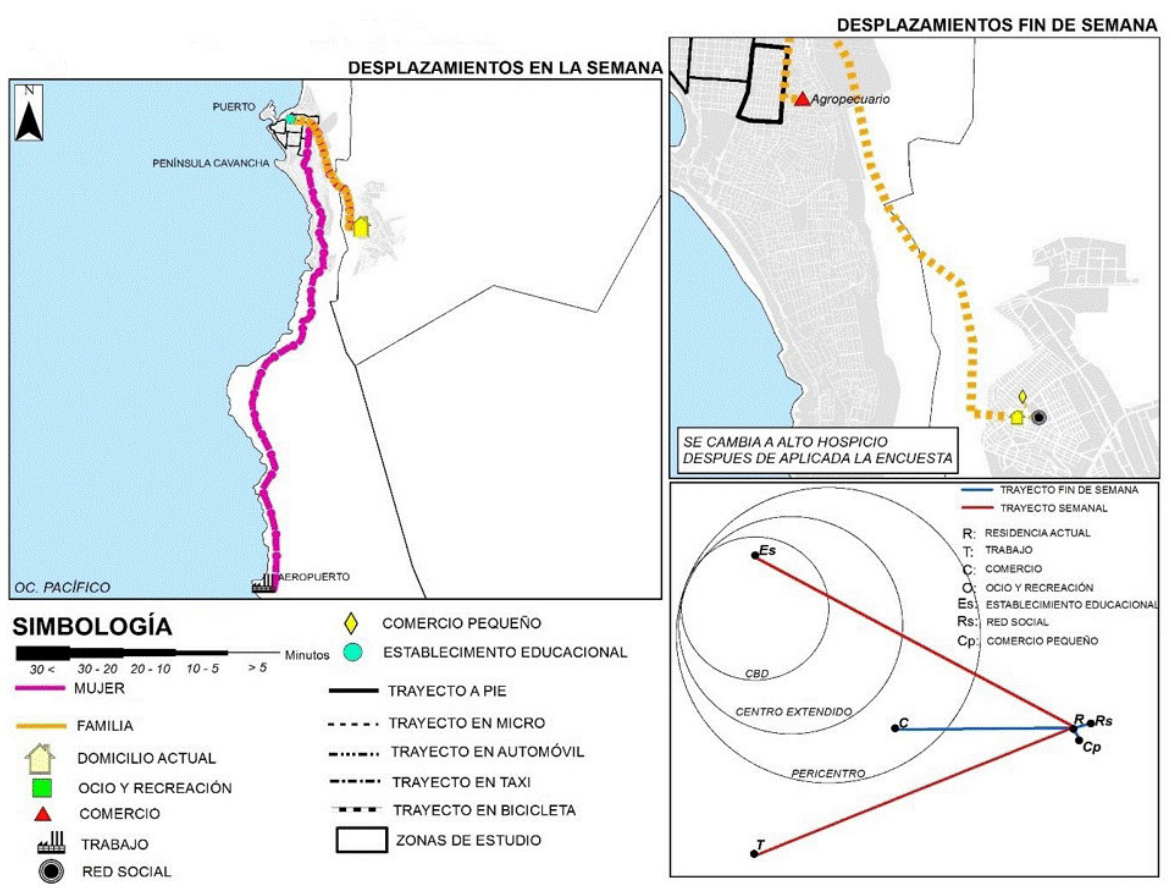

Figura 6. Hogar migrante boliviano 1.2, incierto. Fuente: Elaboración propia a partir de las entrevistas en profundidad Fondecyt $N^{\circ} 11121241$.

El acceso a una vivienda de tamaño mayor a las cuales acostumbraban a arrendar en el área central, permitió la llegada de familiares y amigos, sosteniendo así procesos de tugurización en busca de disminuir los costos de arriendo del hogar, esto funciona como una estrategia que permite el ahorro del grupo migrante. Los procesos de tugurización hacen referencia a modos de ocupación de los inmuebles, según lo evidenciado en la ciudad de Iquique se encuentran: 1) tugurización de la vivienda colectiva con su respectiva ocupación y subdivisión 2) el alquiler de piezas, habitándolas más de una familia, y 3) ocupación de terrenos en sectores periféricos, formando tugurios.

$\mathrm{Al}$ respecto, Mac Donald (2011:6) plantea que "los tugurios no son vistos como un problema por el contrario son vistos como un camino para acceder a la tierra y a la vivienda en contextos donde no es posible hacerlo de otra forma". Por esto existen hogares migrantes que están dispuestos a soportar y transar seguridad, salubridad y calidad habitacional por obtener y mantener una buena accesibilidad ya sea al trabajo, a las redes, al ocio, a la recreación, al servicio, etc. 
Con respecto a las prácticas espaciales de los migrantes, estas responderían a una movilidad sedentaria en la perspectiva de Montulet (2004) en Contreras (2012:350), ya que "todas las experiencias y prácticas urbanas se realizan en referencia a un lugar específico, que sería el barrio y el centro habitado próximo a la residencia". Generalmente esos migrantes se desplazan diariamente en el mismo espacio y con trayectorias cotidianas recurrentes (Ibíd.) debido a que en este confinamiento se resuelven las necesidades básicas para sostener el proyecto migratorio.

Con esto se quiere confirmar y apostar que, en la centralidad la satisfacción de sus prácticas espaciales especialmente en el acceso a los lugares de ocio, recreación y compras disminuye de alguna forma la condición de inequidad y vulnerabilidad en la que se encuentran. Wirth (1921) en Contreras (2012) plantea que el "espacio invertido por una comunidad sirve o actúa como una clase de trampolín para la integración para los recién llegados" (Félonneau, Marchand y Fleury- Bahi, 2005: 116 en Contreras, 2012).

Es importante aclarar que las prácticas espaciales son diferenciadas entre hombres y mujeres, como evidencia la literatura referente a la movilidad femenina (Contreras y Lazo, 2009), sin embargo, en el caso de los hogares entrevistados corresponden a familias, por tanto, han acoplado sus prácticas en función de reducir el espacio-tiempo, por lo cual no se evidencian diferencias sustantivas entre sus recorridos.

\section{Espacios de reserva al migrante}

Lo interesante, además, es que la mayoría de los migrantes ocupan y habitan los mismos espacios donde en otras épocas habitaron segmentos de bajos ingresos constituidos por móviles internos provenientes del sur del país como de la Pampa y migrantes europeos y de origen asiáticos. Estos últimos fueron generando lo que se ha llamado 'enclaves étnicos' (Marcuse, 2001), que refiere a grupos de diversas razas, etnias o nacionalidades que deciden vivir juntos por voluntad propia. Áreas que funcionan como "espacios de reciclaje" o "zonas de transición" (como los llama la Escuela de Chicago) para los nuevos migrantes.

Estas zonas se caracterizan por alojar a población migrante que "sólo se trasladaron cuando pudieran permitirse el lujo de rechazar su deteriorado ambiente o cuando el crecimiento urbano los forzara alejarse aún más" (Hannerz, 1993:39, en Margarit y Bijit, 2014:22). En este mismo sentido (Veiga, 1999 en Margarit y Bijit, 2014) agrega que debieran llamarse áreas de transición, las que se caracterizan por ser lugares con vivienda muy deteriorada, con una gran carencia de servicios. Son áreas que frecuentemente están a la espera de ser remodeladas.

Es así como por ejemplo el cuadrante entre las calles Tarapacá, Thompson, Baquedano y Juan Martínez que a principios del siglo XX concentraban los burdeles de la época, el denominado barrio rojo, hoy ha sido ocupado por panaderías y restaurantes asociadas a la migración peruana, centros de llamados e internet asociados a la migración boliviana y peluquerías donde colombianos y ecuatorianos han podido enlazar trabajo, residencia y redes (Figura 7).

En el caso del cuadrante entre las calles Sotomayor, Esmeralda, Eleuterio Ramírez y Juan Martínez, se concentra la economía netamente boliviana (enclave étnico). Centros de llamados, restaurantes y pensiones son el punto de partida para el inicio en la línea de vida de un migrante boliviano. 
Este espacio responde más bien a la transitoriedad de la migración debido a la existencia de un comercio generado por una migración circular (Bovenkerk, 1974 en Castillo, 1997), es decir, transfronteriza. Es en esta área donde transitan permanentemente, transformando los espacios a medida que estabilizan su proyecto migratorio, a través del acceso a la vivienda, al trabajo, y la integración socio-política.

Es aquí donde la circulación de información permite el sustento de la migración generando redes de apoyo, lo que permite una disminución en la vulnerabilidad que se encuentra el migrante. Estas mismas redes junto a otros factores son los que a migrantes no solo latinoamericanos, sino también provenientes de otros países, influyen en las elecciones que toman, a través de la línea de vida, aunque Stefoni (2014) reconoce que a pesar que las redes disminuyen una serie de costos asociados al proyecto migratorio, estas funcionan para bien y mal, ya que presentan estructuras de poder interna, orden de género y clase que operan en el lugar de destino y origen, lo que permite una serie de accesos a beneficios pero que también restringen a cierta parte de la población a esos mismos beneficios, es decir, son importantes, ya que facilitan pero también limitan.

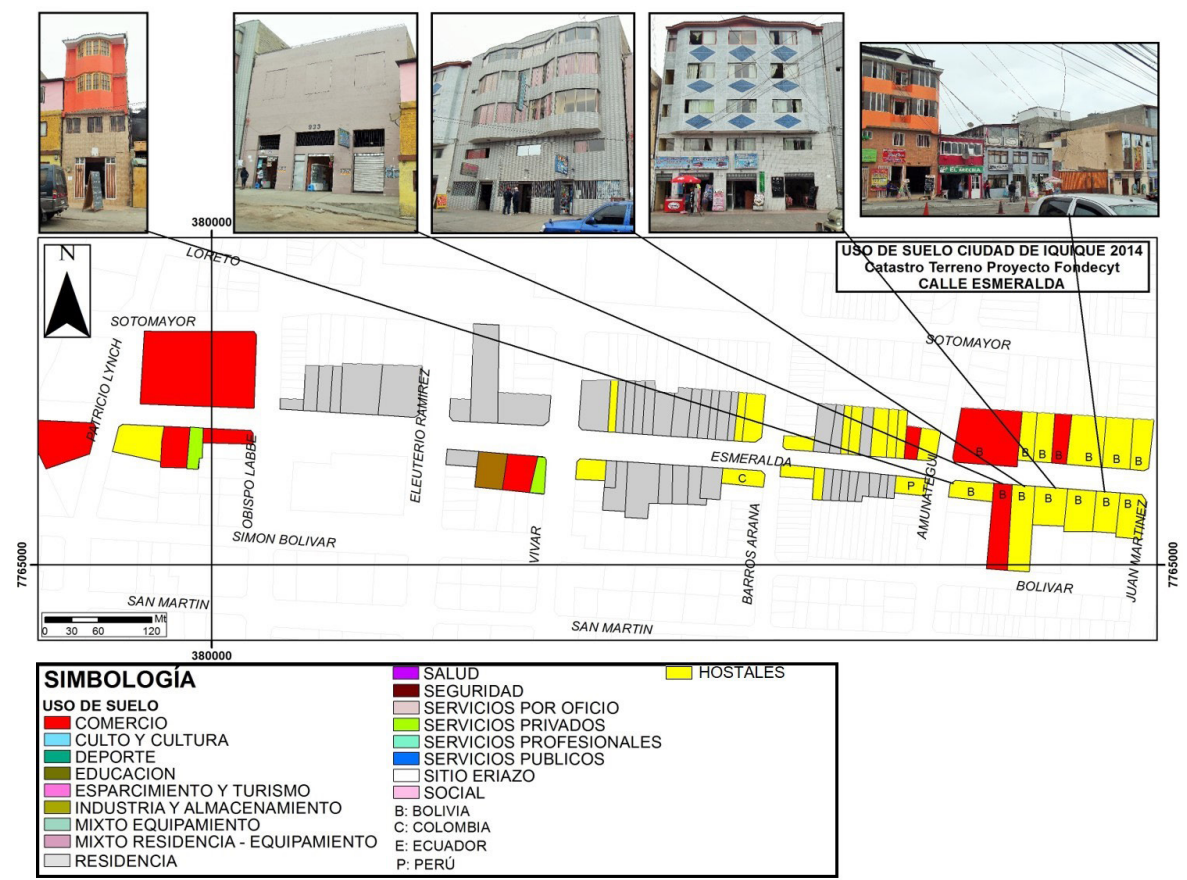

Figura 7. Uso de suelo calle Esmeralda “Área de concentración boliviana”. Fuente: Elaboración propia a partir de las entrevistas en profundidad Fondecyt $N^{\circ} 11121241$.

\section{Elección residencial y transformación del espacio transitado}

Uno de los elementos relevantes referido a la movilidad de los migrantes son las prácticas espaciales generadas por cada entrevistado. Se plantea que estas prácticas espaciales se encuentran fundidas sobre el motivo de elección residencial, las cuales transforman el espacio transitado como también índice sobre el tipo, modo y tiempo de desplazamientos. Por ende, la residencia, como el barrio y/o población funcionan como anclas donde el migrante articula distintas movilidades cotidianas.

$\mathrm{Al}$ analizar el motivo de elección cruzado con la localización y las prácticas espaciales se logró evidenciar que existe una relación estrecha. Cuando el motivo de elección 
residencial es la cercanía al trabajo se observó que el tiempo y la distancia del desplazamiento es menor en una ciudad en que el centro concentra una gran cantidad de flujos por motivos laborales, educacionales y comerciales, mientras que cuando la elección pasa por la cercanía a las redes de contacto, este tiempo y distancia tiende aumentar. Si bien este patrón varía dependiendo de cada caso, ya que en algunos coincide la cercanía del trabajo con la residencia y las redes, se observa que estos son mínimos.

Esta tendencia cambia si se analiza los desplazamientos cotidianos con motivos de compras y ocio, ya que se exhiben prácticas comunes en los migrantes las cuales están asociados a la proximidad. Estos tipos de desplazamientos en cierta medida son los que más se generan en la ciudad de Iquique y son los de mayor importancia.

El uso de la movilidad por proximidad es utilizado como capital de movilidad por los migrantes que tienen como objetivo reducir los costos en términos de tiempos y coste de traslado, ya sea hacia las compras, los servicios y las redes, por ende, la proximidad surge como un recurso que permite la conexión con el barrio y/o población, es decir, la vida cotidiana de los habitantes migrantes se organiza en el marco de unas "cuencas de vida" término acuñado por Dureau, Goueset y Le Roux (2012), estando su uso limitado a los recursos económicos de estos y al potencial restringido de movilidad, para ello se utiliza el término generado Montulet (1998) "movilidad sedentaria", ya que las prácticas espaciales se realizan en un lugar específico. Estas prácticas de proximidad se encuentran limitadas por la configuración de la ciudad debido a que solo ocurren en el área central, el cual funciona como polo atracción de flujos.

Por el contrario, al dirigir los flujos hacia el sector sur de Iquique el tiempo y la distancia de los desplazamientos se ven afectados debido a la geografía de la urbe, que repercuten en la economía del hogar. Para evitar esto, los migrantes recurren a las cuencas de vida explicitadas anteriormente donde el capital de movilidad se ve favorecido.

En lo que se refiere a la transformación de los espacios transitados y habitados, esta tiene relación con nuevamente la etapa del ciclo en que se encuentre el migrante y con las aspiraciones personales llevadas a cabo en el proyecto migratorio. "El espacio vivido de los inmigrantes ha logrado dar construcción a una ciudad distinta, multicultural, llena de espacios resignificados que han contribuido a la transformación física y cultural" (Granados y Yáñez, 2011:4).

En estos espacios se replican las prácticas traídas desde el país de origen, además son puntos de reunión donde las redes cumplen una función importante ya que lograron consolidar la transformación asociada a la estabilidad del grupo de migrante. Además, son espacios donde el comercio, la residencia y otras esferas de la vida permiten cierta mixtura de nacionalidades las cuales comparten en algunos casos proyectos migratorios similares.

\section{Conclusiones}

El motivo de la elección residencial de los hogares migrantes en la ciudad de Iquique, teóricamente ocurre debido a la existencia de una oferta de vivienda antigua representada en viviendas amplias, deterioradas con gran número de habitaciones posibles de subarrendar, que generan tugurios y viviendas insalubres. Pero de las entrevistas se proyectan múltiples factores a la hora de escoger la residencia, los cuales se lograron tipificar como la cercanía al área de servicios, al trabajo y especialmente a las redes. 
Las entrevistas entregaron información sobre los tiempos de desplazamientos en la ciudad de Iquique las cuales permitieron comprobar parcialmente lo siguiente:

La configuración de la ciudad es una limitante a la hora del desplazamiento tanto por motivos laborales u otros afectando el tiempo y distancia de este cuando el commuting debe realizarse hacia y desde fuera de la ciudad como por ejemplo es el caso de los flujos proveniente de Alto Hospicio o comunas interiores se intensificarían los tiempos y distancias de traslado.

Los migrantes que escogen vivir cercanos al trabajo no presentan dificultades en tiempo ni distancia del commuting, debido a que la fuente laboral se encuentra en el centro de la ciudad lo cual permite una comunicación más fluida con la residencia central y con los espacios frecuentados.

$\mathrm{Al}$ escoger vivir cercanos a las redes sociales y familiares los migrantes pueden sufrir problemas con el tiempo y distancia del desplazamiento hacia el trabajo.

Aquellos que conviven próximos a las redes, y que coinciden con el trabajo, tienden a modificar el espacio habitado, transformando la cara del barrio.

Las redes sociales siguen siendo importantes en el hallazgo no solo de los actuales espacios de residencia, aunque éstas también enfrentan transformaciones, sino también sirven en el hallazgo de trabajo, ayuda legal, sostén cultural, perpetuación del movimiento, etc.

ACLARACIÓN. Esta pesquisa es parte de los resultados obtenidos en el trabajo denominado "Trayectorias Residenciales y prácticas espaciales de los hogares migrantes latinoamericanos del centro y peri centro de la ciudad de Iquique", enmarcada en el Proyecto FONDECYT N 11121241 de la investigadora Yasna Contreras, del Depto. de Geografía de la Facultad de Arquitectura y Urbanismo de la Universidad de Chile 


\section{Q Bibliografía}

»Castillo,J.(1997). Teorías de la migración de retorno, OIM Organización Internacional para las Migraciones, Universidad de Complutense, mimeo, Madrid, España. <"http://ruc.udc.es/dspace/bits-tream/2183/9664/1/CC_33_art_3.pdf\%20" http://ruc.udc.es/dspace/bits-tream/2183/9664/1/CC_33_art_3.pdf >.

»Contreras, Y. (2012). Cambios socio-espaciales en el centro de Santiago de Chile: Formas de anclarse y prácticas urbanas de los nuevos habitantes. Universidad Católica de Chile. Tesis para obtención del grado de doctor de Arquitectura y Estudios Urbanos.

»Córdoba, H. (2014). Mobilité internationale et dynamiques résidentielles à Bogotá (Colombie). Thèse de Doctorat, Université Européenne de Bretagne, Université de Rennes 2.

" Dubucs, H. (2009). Habiter une ville lointaine. Tesis de doctorado para optar al título de Doctor en Geografía, Départament de Géographie, Université de Poitiers.

"Dureau, F. (2007). Bogotá: una doble dinámica de expansión espacial y de densificación de espacios ya urbanizados. En F. Dureau, V. Dupont, E. Leliéfvre, J. Lévy, \& T. Lulle, Metrópolis en movimiento, una comparación internacional. Bogotá: Alfaomega Colombiana.

"Dureau, F., Goueset, V., y Le Roux, G. (2012). Movilidad cotidiana, prácticas del espacio urbano y desigualdades socioterritoriales en dos periferias populares de Bogotá: Soacha y Madrid (1993 - 2009). X seminario ACIUR, Universidad Javeriana, Mesa Temática 18, 1-21. Colombia

»Dupont, V., y Dureau, F. (1994). Rôle des mobilités circulaires dans les dynamiques urbaines. Ilustrations à partir de l'Équater et de l'Inde. TiersMonde, 801-829.

» Garzón, L (2010). Estudio de las dinámicas de movilidad residencial y su efecto en el uso del suelo del área metropolitana del valle del Aburrá. Medellín: Universidad Nacional de Colombia.

"Granados, S. (2011). Línea de vida de un inmigrante Latinoamericano: Desde la decisión hasta la transformación en la ciudad de Santiago de Chile. Revista Geográfica de América Central (EGAL 2011), 1-15.

" Granados, S., y Yáñez, G. (2011). Inmigrantes como entes transformadores de la ciudad: Latinoamericanos en Santiago de Chile. Documento no publicado

»Guerrero, B. (2007). La ciudad y sus transformaciones: Memoria Urbana de lquique. Revista de Ciencias Sociales, (19), 149-165.

»Hiernaux, D., y Lindón, A. (2006). Tratado de Geografía Humana. Barcelona: Anthropos.

» Instituto Nacional de Estadísticas; INE. (2012). Resultados preliminares censo de población y vivienda.

" Jiménez, M. (2009). Potencialidades de la medición de la movilidad cotidiana a través de los censos. Seminario-taller. Los censos de 2010 y la migración interna, internacional y otras formas de movilidad territorial.

"Lévy, J-P., y Dureau, F. (1998). La mobilité residentiel dans ke douaisis lille Francia. Observatoire regional de l'habitat et de l'amenagement. 
"Liberona, N. (2015). De las fronteras geopolíticas a las fronteras sociales. La migración boliviana a través de la prensa de Tarapacá (1990-2007). Revista Estudios Fronterizos. 16, (32), 41-74.

"Llano, C. (2005). Localización residencial y movilidad laboral: un análisis del commuting de trabajadores nacionales $e$ inmigrantes en la comunidad de Madrid. Universidad Autónoma de Madrid, Facultad de Ciencias Económicas, Departamento de Análisis Económico: Teoría Económica e H. Económica, Madrid.

" Mac Donald, J. (noviembre de 2011). Ciudad, pobreza, tugurio. Aportes de los pobres a la construcción del habitad popular. Obtenido de Habitad y Sociedad: www.habitadysociedad.us.es

" Marcadet, Y. (2007). Habitar en el centro de la Ciudad de México. Prácticas espaciales en la Santa María La Ribera. Alteridades, 17(34), 39-55

" Marcuse, P. (2001). Enclaves Yes, Ghettoes, No: Segregation and the State. Lincoln Institute of Land Policy Conference Paper.

» Margarit, D., y BIJIT, K. (2014). Barrios y población inmigrantes: el caso de la comuna de Santiago. Revista INVI, 29, (81), 19-77.

» Montulet, B. (1998). Les enjeux spatio temporels du social. París: L'Harmattan.

» Ortiz, J., y Schiappacasse, P. (1997). Las migraciones intraurbanas y su impacto en la diferenciación del espacio social del Gran Santiago. Revista Geográfica de Chile Terra Australis, 42, 121- 138.

"Palma, P. (2014). Trayectorias residenciales y prácticas espaciales de los hogares migrantes latinoamericanos del centro y pericentro de la ciudad de lquique. Tesis para optar al título de Geógrafo, Escuela de Geografía, Universidad de Chile.

»Rodríguez, J. (2008). Movilidad cotidiana, desigualdad social y segregación residencial en cuatro metrópolis de América Latina. Revista Eure, XXXIV, 49-71.

»Stefoni, C. (2002). Inmigración peruana en Chile. Una oportunidad para la integración. Santiago de Chile: Editorial Universitaria.

»Stefoni, C. (5 de agosto de 2017). Migración Latinoamericana. (P. P. Calorio, Entrevistador).

» Tapia, M. (2012). Frontera y migración en el norte de Chile a partir del análisis de los censos de población. Siglos XIX- XXI. Revista de Geografía Norte Grande, 53, 177-198.

\section{Pedro Palma Calorio / ppalma@observatoriocite.cl}

Geógrafo por la Universidad de Chile. Magister de Desarrollo Urbano del Instituto de Estudios Urbanos de la Pontificia Universidad Católica de Chile. Co-fundador de la ONG Observatorio CITé.

\section{Gricel Labbé Céspedes / glabbe@observatoriocite.cl}

Geógrafa por la Universidad de Chile. Diplomada en Derechos Humanos, Derecho a la vivienda y Políticas Públicas de la Fundación Henry Dunant y, Magister de Desarrollo Urbano del Instituto de Estudios Urbanos de la Pontificia Universidad Católica de Chile. Directora de la ONG Observatorio CITé. 